\title{
An in vitro technique to identify the RNA binding-site sequences for RNA-binding proteins
}

\author{
SunKyung Choi ${ }^{1}$, Chungoo Park², Kyoon Eon Kim¹ ${ }^{1}$, and Kee K. Kim ${ }^{1}$ \\ ${ }^{1}$ Department of Biochemistry, College of Natural Sciences, Chungnam National University, Daejeon, \\ Republic of Korea and 2School of Biological Sciences and Technology, Chonnam National University, \\ Gwangju, Republic of Korea
}

BioTechniques 62:28-33 (July 2017) doi 10.2144/000114567

Keywords: RNA-binding protein (RBP); RBFOX2; FUS; SAM68; SFPQ

Supplementary material for this article is available at www.BioTechniques.com/article/114567.

RNA-protein interactions play a major role in gene regulation. Although many techniques to analyze RNA-protein interactions have been developed, noteworthy challenges such as determining the RNA sequences that bind RNA-binding proteins (RBPs) remain unsolved. Here, we describe a novel technique using a 4-thio-uridine-incorporated RNA pool to identify the RBP-binding consensus sequences for RBPs produced by in vitro transcription and translation. To confirm the fidelity of this approach, we determined the consensus RBP-binding sequence for RBFOX2, UGC(A/U)(A/U)NU, which is very similar to the known RBFOX2-binding sequence, UGCAUG. Using our method, consensus RBP-binding sequences were determined for three RBPs, namely FUS (fused in sarcoma), SFPQ (splicing factor proline and glutamine rich), and SAM68 (Src-Associated substrate in Mitosis 68 kDa). The consensus RBP-binding sequences for these RBPs were confirmed by RNA-protein complex immunoprecipitation-PCR analysis.

RNA-binding proteins (RBPs) are proteins that bind to single- or double-stranded RNA to form ribonucleoprotein (RNP) complexes, which play important roles in regulating post-transcriptional processes such as splicing, polyadenylation, capping, modification, mRNA stabilization, and mRNA localization (1-4). Therefore, RBPs are extensively involved in processes ranging from development to stress responses. Abnormal expression of RBPs affects many stages of RNA metabolic processing and alters gene expression patterns. Given that changes in RBP expression patterns and functional abnormalities are related to many diseases, such as cancer, identification of the properties of RBPs involved in RNA interactions is essential to studies of RNA regulatory processes and related diseases. While the importance of RBPs has been recognized, and several types of RBPs have been identified in various organisms, including plants, animals, and microbial species (5), many RBP functions have yet to be defined, and there is insufficient information on the interactions between RNA and RBPs, which are essential to identify their functions.

For analysis of RNA-RBP interactions, systematic evolution of ligands by exponential enrichment (SELEX) and cross-linking immunoprecipitation (CLIP) have been used to determine the consensus sequences for RNA binding to RBPs. SELEX involves the construction of a randomly generated nucleic acid library, followed by binding of the library to the target ligand $(6,7)$. A partitioning process is used to select and isolate sequences with high binding capacity, and these are then amplified using PCR. Multiple cycles of this selection-amplification process increase the proportion of nucleic acid sequences within the pool that bind the target ligand, and the final stage of selection yields sequences with high affinity for the target ligand. The amplified DNA is then cloned, and its nucleotide sequence is analyzed. However, the size of the library is limited, it is difficult to determine the optimal conditions for selection, and repeating multiple cycles is time-consuming $(8,9)$.

CLIP involves cross-linking of target RNA and proteins, followed by immunoprecipitation of RNA-bound protein complexes using a specific antibody in order to select the RNA fragment bound to the RBP (10-12). Types of CLIP include photoactivatable-ribonucleoside-enhanced CLIP (PAR-CLIP), high-throughput sequencing of RNA isolated by CLIP (HITS-CLIP), and individual nucleotide resolution CLIP (iCLIP) (13-15). All current CLIP methods require relatively large amounts of cells or tissue to obtain libraries that contain the transcriptome (16). Moreover, all CLIPbased experimental methods suffer from the possible selection of highly expressed

\section{METHOD SUMMARY}

We developed a novel technique wherein a 4-thio-uridine-incorporated RNA pool is used to identify the consensus RNA binding site sequences for RNA binding proteins (RBPs) produced by in vitro transcription and translation. 


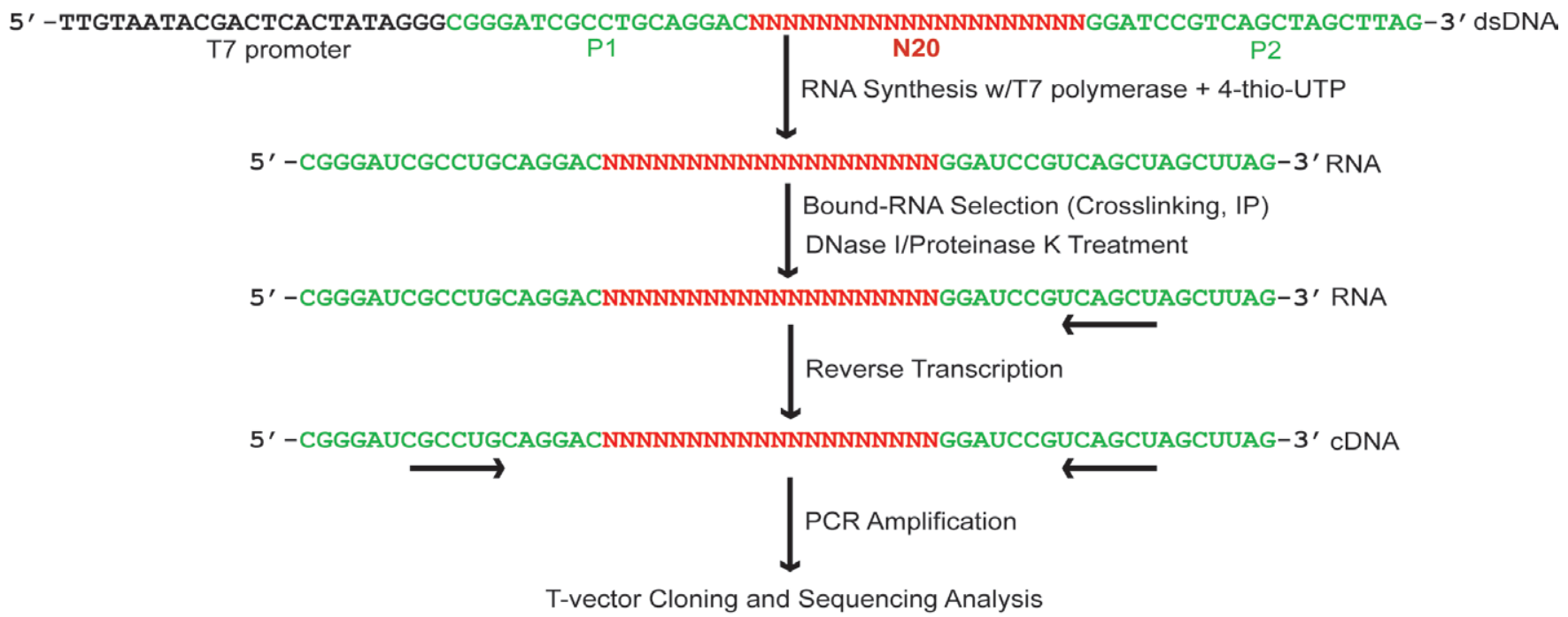

Figure 1. Schematic illustration of the selection strategy for RNA sequences binding RNA-binding proteins (RBPs). N20 (red nucleotides) indicates the random region. P1 and P2 (green nucleotides) indicate the primer positions for PCR amplification.

non-target RNAs (despite their low binding affinity), as well as reduced selection of poorly expressed target RNAs (despite their high binding affinity).

Recently, various improved methods have been developed to identify RBP-binding consensus sequences; however, most of these methods are limited to mapping and analyzing data sets. Furthermore, alternatives are required to overcome the limitation of selection based on RNA abundance, rather than on binding affinity. Here, we report a new RNA SELEX method using a 4-thio-uridine-incorporated RNA pool that does not depend on target RNA abundance and does not require large amounts of cells or tissue. RBFOX2 (RNA-binding protein, fox-1 homolog 2), a well-characterized RBP with a known consensus sequence, was used to validate this new technique. The technique was then used to identify the consensus sequences of the RBPs FUS (fused in sarcoma), SFPQ (splicing factor proline and glutamine rich), and SAM68 (Src-associated substrate in mitosis, $68 \mathrm{kDa}$ ).

\section{Materials and methods}

Oligonucleotide library and preparation of 4-thio-uridine-incorporated RNA pool The oligonucleotide library that was used contains different single-stranded DNA sequences, each with a central random region (20 nucleotides) flanked by constant sequences for primer binding during PCR amplification. The random DNA library was converted to a double-stranded
DNA library by PCR amplification with a T7 promoter site 5'-TTG TAA TAC GAC TCA CTA TAG GG-3'; sense primer 5'-CGG GAT CGC CTG CAG GAC-3' (P1); and antisense primer 5'-CTA AGC TAG CTG ACG GAT CC-3' (P2). A 4-thio-uridine-incorporated RNA pool was synthesized from this double-stranded DNA library by T7 RNA polymerase in the presence of 4-thio-UTP using a MAXIscript kit (Ambion/Thermo Fisher Scientific, Waltham, MA).

\section{Preparation of RBPs and} immunoblot analysis

The full-length coding regions of the RBFOX2 (NM_001031695), FUS (NM_004960), SFPQ (NM_005066.2), and SAM68 (NM_006559.2) cDNAs were introduced into the plasmid pCS3+MT, which contains the T7 promoter and six copies of myc epitopes. These Myc-RBPs were synthesized in vitro from the pCS3+MT constructs using the TNT Coupled Reticulocyte Lysate System (Promega, Madison, WI). Samples were separated on a NuPAGE 4\%-20\% Bis-Tris Gel (Thermo Fisher Scientific), and transferred to a nitrocellulose membrane. The membrane was blocked with PBS containing $5 \%$ skimmed milk, incubated overnight at $4^{\circ} \mathrm{C}$ with anti-myc antibody in the blocking solution, and washed $3 x$ with PBS containing 0.05\% Tween-20. The binding of horseradish peroxidaseconjugated secondary antibody (Abcam, Cambridge, UK) was detected using the SuperSignal system (Thermo Fisher Scientific). Anti-myc antibody (1:5000)
(RRID: R950-25, AB_2556560) was obtained from Thermo Fisher Scientific (Waltham, MA).

\section{RNA-protein complex}

immunoprecipitation and selection Binding of a myc-RBP with the 4-thio uridine-incorporated RNA pool was carried out in a reaction containing 10 mM HEPES, $\mathrm{pH}$ 7.9, 2 mM MgCl, 1 mM ATP, $20 \mathrm{mM}$ creatine phosphate, $50 \mathrm{ng}$ yeast tRNA, 2 mM DTT, and $2 \%$ polyethylene glycol (molecular weight 3550) for 20 min at $30^{\circ} \mathrm{C}$. The reaction mixtures were irradiated with 365-nm UV light for 20 min on ice and then immunoprecipitated with the anti-myc antibody using a Magna RIP kit (EMD Millipore, Billerica, MA) according to the manufacturer's instructions. After DNase I and proteinase $\mathrm{K}$ digestion, the RNA recovered from the complexes was reverse-transcribed using SuperScript III reverse transcriptase (Thermo Fisher Scientific) with the primer 5'-CTA AGC TAG CTG ACG GAT CC-3' (P2). The following PCR primers were used to amplify the cDNAs of the RBP-bound RNA: 5'-CGG GAT CGC CTG CAG GAC-3' (P1) and 5'-CTA AGC TAG CTG ACG GAT CC-3' (P2). The PCR products were then cloned in the TOPO-T vector (Invitrogen) and sequenced.

For the RIP-PCR analysis, oligonucleotides containing RBP-binding sequences (Supplementary Table S1) with a central region (20 nucleotides) flanked by constant sequences for primer binding during PCR amplification and the T7 promoter for RNA transcription were used to synthesize the 
A

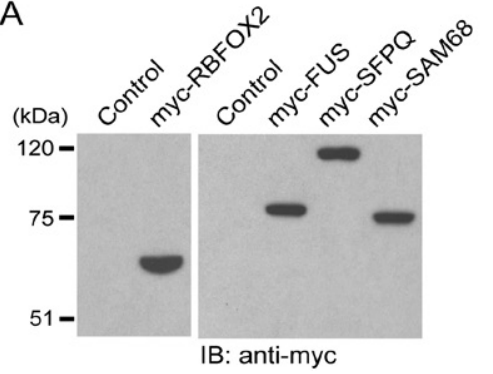

B

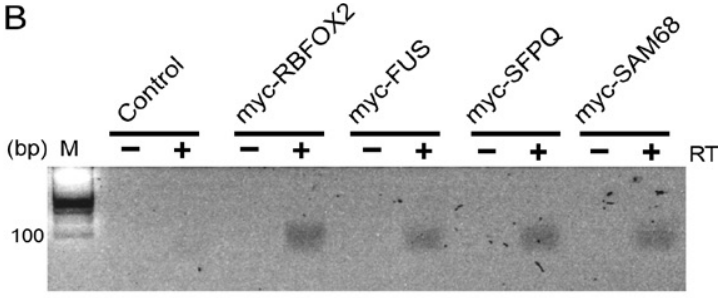

Figure 2. Identification of RNA-binding protein (RBP)-RNA interaction. (A) The indicated myc-tagged RBPs were analyzed by immunoblotting using anti-myc antibody. Rabbit reticulocyte lysate containing the pCS3+MT empty vector was used as a negative control (Control). (B) RT-PCR analysis of the RNAs associated with the indicated RBPS. PCR products after reverse transcription (+); samples without RT (-) served as negative controls for dsDNA contamination. M: marker.

RNAs. The RBP-RNA complexes were immunoprecipitated with the anti-myc antibody without irradiation using a Magna RIP kit according to the manufacturer's instructions. The RNAs recovered from the complexes were subjected to RT-PCR to amplify RBP-binding RNAs. The following PCR primers were used to obtain the cDNAs for the RBP-binding RNAs: 5'-CGG GAT CGC CTG CAG GAC-3' (P1) and 5'-CTA AGC TAG CTG ACG GAT CC-3' (P2). The end products of the RT-PCR were visualized by agarose gel electrophoresis on a $2 \%$ agarose gel.

\section{Binding site analysis}

The Multiple Expression Motifs for Motif Elicitation (MEME) program version 4.11.2 web site (http://meme-suite.org/) was used to identify the consensus sequences. Parameters were set to discover sequences 6-11 nucleotides in length.

\section{Results and discussion}

In vitro technique to identify RNA sequences binding to RBPs

To identify consensus RNA sequences bound by RBPs, an oligonucleotide library consisting of single-stranded DNA sequences with a 20 -nucleotide random region in the center and primer binding sequences at each end for PCR amplification was constructed in vitro (Figure 1). This random oligonucleotide library was amplified by PCR for conversion into a double-stranded DNA library and the addition of a T7 RNA polymerase promoter sequence. T7 RNA polymerase was then used in the presence of 4-thio-UTP, a photoactivatable ribonucleoside analog, to synthesize a 4-thio-uridine-incorporated RNA pool from the double-stranded DNA. This method resulted in a similar abundance of each RNA molecule in the pool, thus ensuring that binding affinity was the only factor influencing the selection. Myc-tagged RBPs were synthesized by in vitro transcription and translation, mixed with the 4-thio-uridine-incorporated RNA pool, and then UV-crosslinked at 365 $\mathrm{nm}$. Use of a photoactivatable ribonucleoside allows for application of higher UV wavelengths, such as $365 \mathrm{~nm}$, compared with the $254 \mathrm{~nm}$ wavelength traditionally used for crosslinking. This further simplifies protein-RNA binding, resulting in 100- to 1000-fold improvements in RNA co-immunoprecipitation efficiency using the same amount of UV radiation energy (13). Crosslinked RNA-protein complexes were immunoprecipitated with the anti-myc antibody and then proteinase K-digested to isolate crosslinked target RNA fragments. Reverse transcription of the isolated RNA was performed with the P2 primer, and PCR amplification was conducted using the P1 and P2 primers. Finally, the PCR product was cloned in the T-vector, and the RNA sequences binding to the RBP were identified by sequence analysis.

Immunoblot analysis using anti-myc antibody was done to confirm the proper synthesis of the myc-tagged RBPs; RBFOX2, FUS, SFPQ, and SAM68 were found at the expected sizes (Figure 2A). To determine whether RNA isolated from the immunoprecipitated RNA-protein complex was contaminated by dsDNA, cDNA synthesized by reverse transcription (RT) and RNA not subjected to RT were amplified by PCR. The PCR products were compared by electrophoresis on a $2 \%$ agarose gel, and the results showed only PCR products for the samples that underwent RT (Figure 2B). To avoid indirect interactions between an RBP and RNAs that were not crosslinked, we performed RNA-protein immunoprecipitation without crosslinking. As expected, no PCR products without UV-crosslinking were observed (data not shown). There were no PCR products in an empty vector control, suggesting that the PCR products are derived from RBP-bound RNAs.

\section{Applications of the technique}

RBFOX2 is a key regulator of alternative splicing and binds to a well-conserved consensus sequence, (U)GCAUG, as confirmed by SELEX and CLIP $(17,18)$. We applied our newly developed method to RBFOX2 and compared the results obtained to a well-known consensus sequence. The MEME program was used to analyze 88 sequences, which were confirmed by sequencing analysis, to determine the consensus RBP-binding sequence for RBFOX2 using our method (Supplementary Table S2 and Figure 3A). The results showed that 13 sequences contained UGC(A/U)(A/U)NU, accounting for $15 \%$ of the total sequences observed. This result is supported by the previously known consensus sequence, UGCAUG, and reflects the high accuracy of our new method. The second consensus sequence identified was $A(U / A) C(G / A) A A(A / C)$ $(A / C) N G$, accounting for $6 \%$ of the total sequences observed.

Using our method, the consensus RBP-binding sequences for FUS and SAM68 were identified and compared with the previously known sequences, while the consensus sequence for SFPQ was newly identified (Supplementary Table 2, Figure 3B-D). FUS binds to a known sequence, GGUG, and encodes the multifunctional protein component of the heterogeneous nuclear ribonucleoprotein complex, which is involved in pre-mRNA splicing and cytoplasmic export of fully processed mRNA (19). Analysis of the consensus RBP-binding sequence for FUS revealed 7 AGN(A/C)(A/G)NNNUG sequences, accounting for $9 \%$ of the 80 sequences observed (Figure 3B). Furthermore, $\mathrm{G}(\mathrm{A} / \mathrm{C})$ $U N(U / C)(G / C) A(A / G) \cup G$ and $G C U(U / G)$ $C(C / G) A(G / C) C$ each accounted for $5 \%$ of the total sequences observed. SFPQ interacts with polypyrimidine tract binding protein 1 (PTBP1), non-POU domaincontaining octamer binding protein (NONO), cell division cycle 5-like protein (CDC5L), and ubiquitin $\mathrm{C}$ and is associated with renal cell carcinoma and papillary and adrenal neuroblastoma through the GPCR signaling and mRNA splicing pathways 
(20-24). The experimental results for SFPQ revealed 10 UAANGGCU(A/G) sequences, accounting for $13 \%$ of the 79 sequences observed (Figure 3C). The second identified consensus sequence was $\mathrm{G}(\mathrm{U} / \mathrm{C}) \mathrm{U}(\mathrm{U} / \mathrm{G})$ GNACCCA, which accounted for $4 \%$ of the total sequences observed. Finally, SAM68 binds to a known sequence, UAAA, and is involved in various cellular processes, such as alternative splicing, cell cycle regulation, RNA 3'-end formation, tumorigenesis, and human immunodeficiency virus gene expression (25). The experimental results for SAM68 showed $5 \mathrm{GU}(\mathrm{G} / \mathrm{C}) \mathrm{CN}(\mathrm{G} / \mathrm{C})$ $(\mathrm{G} / \mathrm{C})(\mathrm{C} / \mathrm{A})(\mathrm{G} / \mathrm{C}) \cup$ sequences, accounting for $6 \%$ of the 91 sequences observed, whereas the sequence $A(G / C) A A G G$ accounted for 3\% (Figure 3D) of the total sequences.

\section{Validation of the technique}

Although the RBFOX2 consensus binding sequence determined in this study was highly similar to the known binding sequence, the consensus binding sequences obtained for the other RBPs differed from previously identified RNA sequences. To further examine the importance of the consensus RNA sequences determined in this study, we used RNA-protein co-immunoprecipitation-PCR to compare the binding of the RBPs to the RNAs and to RNAs mutated in their RBP-binding motifs (Figure 4). There were two copies of the consensus RNA sequences within the test RNAs. RBFOX2 bound more strongly to the consensus RNA sequences obtained in this study than to the known RBFOX2-binding consensus sequence (Figure $4 \mathrm{~A}$ ). Mutation of the RBFOX2-binding consensus sequence abolished RBFOX2 binding. A previous study showed that FUS binds to GGUGcontaining RNAs (26). Stronger binding by FUS to the consensus RNA sequences obtained in this study than to the GGUGcontaining RNA was observed, and this binding was clearly reduced by transversion mutations in the FUS-binding RNA sequences (Figure 4B). The SFPQ consensus sequence was not identified previously. Binding of SFPQ to the consensus RNA sequences obtained in this study was observed by RIP-PCR, and this binding was clearly reduced by mutation (Figure 4C). The SAM68 consensus sequence was identified as $U(U / A) A A$ by SELEX (27). Binding of SAM68 to UAAA RNA was weaker than to the consensus RNA sequence obtained by our method

A

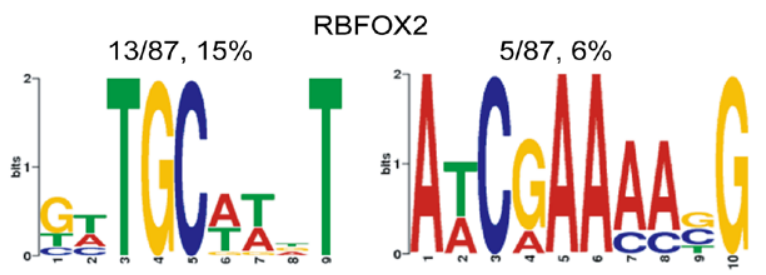

B

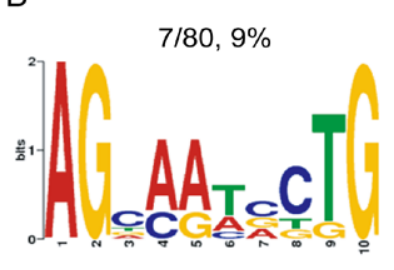

FUS
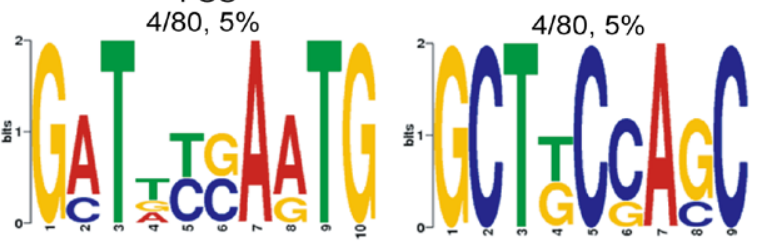

C

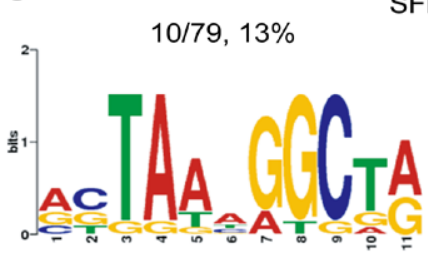

SFPQ
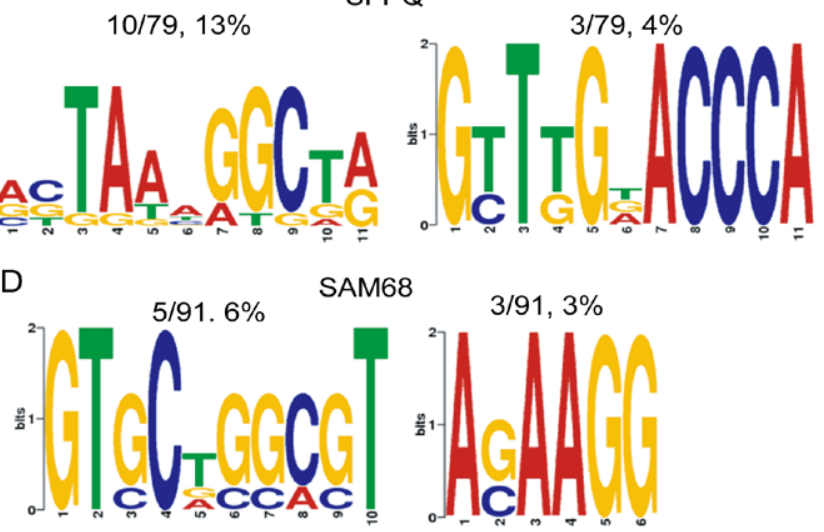

Figure 3. Identification of RNA consensus sequences for RNA-binding proteins (RBPs). The sequence logo represents the consensus sequence discovered by MEME version 4.11.2 analysis of experimentally determined RBP-binding sequences.

(Figure 4D). The binding of SAM68 to the consensus RNA sequences was clearly reduced by transversion mutations in the SAM68 binding sequences. These results demonstrate that binding of the RBPs to the RNA consensus sequences obtained in this study is specific.

Here, we developed a novel technique in which a pool of 4-thio-uridine-incorporated random RNA sequences was used to identify the consensus RNA sequences for binding RBPs. This technique was applied to identify the consensus RBP-binding sequences for RBFOX2, FUS, SFPQ, and SAM68. Moreover, we confirmed the RBP-binding activity of the consensus RNA sequences obtained in this study and determined the fidelity of this technique for identifying RBP-binding consensus sequences.

SELEX was previously carried out using purified protein from Escherichia coli to identify the consensus binding sequences for RBPs. Although the efficiency of protein synthesis in $E$. coli is high, this bacterium cannot carry out post-translational modifications, such as glycosylation, for mammalian proteins because the cellular machinery used for glycosylation in mammals differs from that in $E$. coli. In addition, since there is no endogenous membrane structure that synthesizes integral membrane proteins and only native prokaryotic chaperones may exist, eukaryotic proteins may not fold correctly inside a bacterial cell in some cases. Because reticulocytes contain a native system for mammalian protein synthesis, eukaryotic cell-specific posttranslational processing such as ubiquitinconjugation, $\mathrm{N}$-myristoylation, phosphorylation, isoprenylation, acetylation, signal peptide processing, and core glycosylation are possible. Protein post-translational processing, including modifications and folding, have been shown to influence protein function in eukaryotes (28). Therefore, protein post-translational processing of RBPs may affect their RNA-binding specificities. In this study, rabbit reticulocyte lysate was used rather than $E$. coli for protein 
A
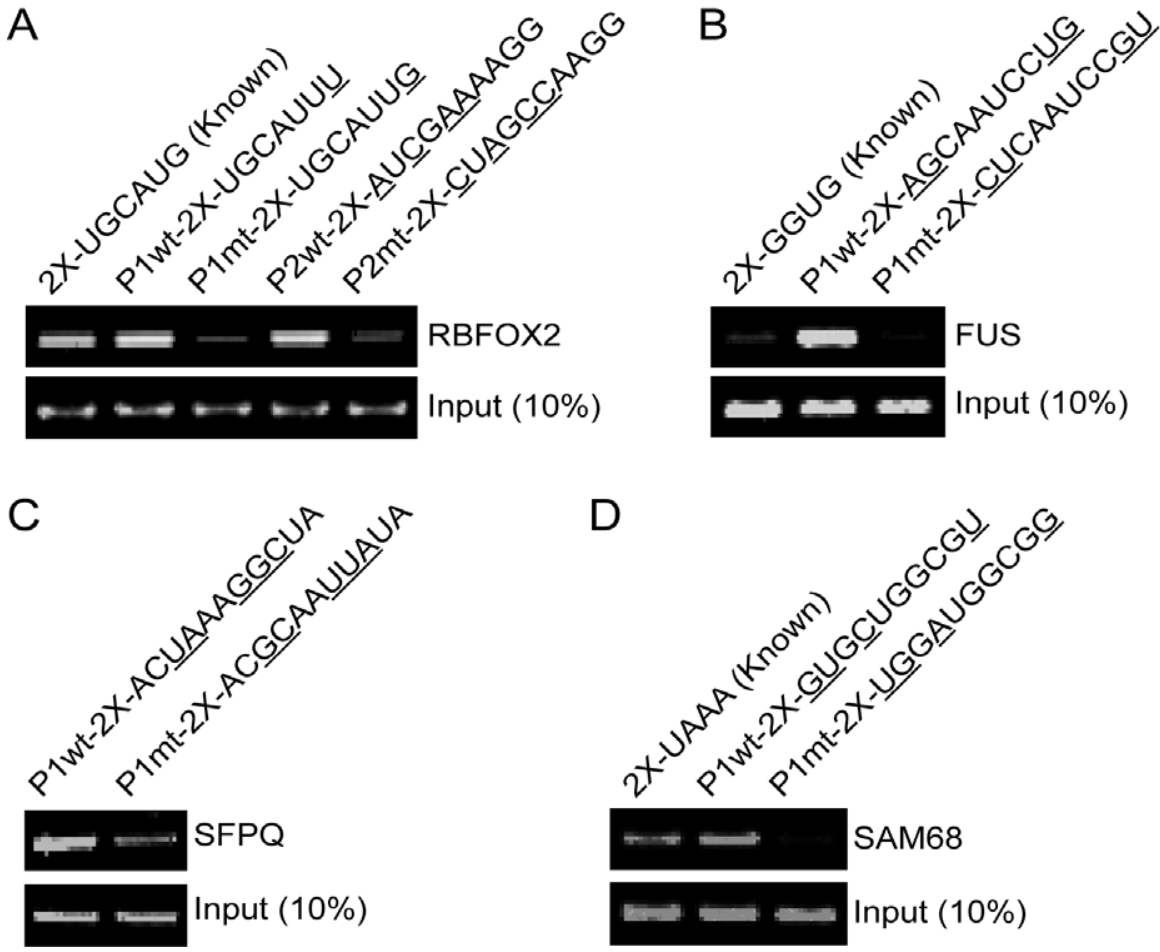

Figure 4. Validation of consensus RNA sequences for RNA binding proteins (RBPs). RNA-protein co-immunoprecipitation (RIP) was carried out with the indicated RNAs and in vitro-synthesized myc-RBPs using the anti-myc antibody. RNAs associated with each RBP were analyzed by RT-PCR. The end products of RT-PCR were visualized by agarose gel electrophoresis. The presence of equal amounts of RNA among a set of binding reactions was verified using in vitro-synthesized RNA without immunoprecipitation (input). Underlined portions of the RNA sequences indicate the mutated sequence ( $\mathrm{mt}$ ) compared with the wild-type sequence (wt). "Known" indicates the known RBP-binding sequence from a previous report (Supplementary Table S1).

synthesis to determine the precise binding site of RBPs in vitro.

SELEX is not quantitative because it requires many rounds of $\mathrm{PCR}$ amplification and is biased toward the strongest binding motif. Because CLIP utilizes cells and tissues, it is restricted to specific cell types and contacts formed at specific time points. In addition, when a specific RNA sequence is expressed in large amounts, it is easy to select for even if its binding affinity for the RBP is low, whereas if its abundance is low, selection is more difficult even if its binding affinity for the RBP is high. Therefore, in this study, the disadvantages of SELEX and CLIP were overcome by our new technique. Because we use a random oligonucleotide library instead of RNA from cells and tissues, the sequences in the RNA pool had similar abundances, overcoming the disadvantage of conventional methods such as CLIP, which is influenced by the abundances of the target RNA sequences. In addition, our technique can be used to identify the overall range of binding motifs, from strong binding motifs to relatively lower-strength binding motifs. Occasionally, low-strength binding motifs are important. Therefore, when determining the consensus RNA sequence for binding a particular RBP, the overall range of binding motifs should be identified.

To overcome the limitations of conventional methods for identifying the consensus binding sequences for an RBP, several methods have been developed and refined, including RNA mechanically induced trapping of molecular interactions, RNAcompete, RNA-MaP (quantitative analysis of RNA on a massively parallel array), and RNA Bind-n-Seq (29-32). RNA mechanically induced trapping of molecular interactions quantifies the binding of a single protein for hundreds of RNA species using microfluidics and microarrays and is applicable when the RNA target is already known. RNAcompete uses fluorescently labeled protein-bound RNA and microarray analysis. Because this method uses sequences no more than $\sim 8$ nucleotides long in the initial RNA library, it may reveal shorter motifs than other methods. In addition, the number of probes analyzed and the commonly used low temperature makes it difficult to analyze the effect of RNA secondary structure on RNA binding. RNA-MaP uses a highthroughput sequencer to measure the binding of fluorescently labeled proteins to RNA. However, it includes a next-generation sequencing (NGS) step, which is relatively costly. RNA Bind-n-seq is a method optimized for analysis over different affinity ranges using different $\mathrm{RBP}$ concentrations, but it is as costly as RNA-MaP because of the NGS step.

In conclusion, using a 4-thio-uridineincorporated RNA pool to identify an RNA consensus sequence that binds to an RBP showed significant accuracy, as observed in experiments using RBFOX2. Furthermore, the limitations of methods affected by target RNA abundance, as in CLIP, were overcome by this method, and the consensus sequence was identified based on the binding affinity. This allowed the method reported in this study to analyze the consensus RNA sequences for binding RBPs such as FUS, SFPQ, and SAM68 in order to identify binding sites within physiological RNA targets.

\section{Author contributions}

S.K.C., C.P., K.E.K., and K.K.K. conceived and designed the experiments. S.K.C., C.P., and K.K.K. performed the experiments. S.K.C., C.P., K.E.K., and K.K.K. analyzed the data. S.K.C. and K.K.K. wrote the paper.

\section{Acknowledgments}

This work was supported by the National Research Foundation of Korea within the Basic Science Research Program through the Ministry of Education under Grant NRF-2015R1C1A1A02036324.

\section{Competing interests}

The authors declare no competing interests.

\section{References}

1. Halbeisen, R.E., A. Galgano, T. Scherrer, and A.P. Gerber. 2008. Post-transcriptional gene regulation: from genome-wide studies to principles. Cell. Mol. Life Sci. 65:798-813.

2. Hieronymus, H. and P.A. Silver. 2004. A systems view of mRNP biology. Genes Dev. 18:2845-2860.

3. Keene, J.D. 2007. RNA regulons: coordination of post-transcriptional events. Nat. Rev. Genet. 8:533543.

4. Keene, J.D. and S.A. Tenenbaum. 2002. Eukaryotic mRNPs may represent posttranscriptional operons. Mol. Cell 9:1161-1167.

5. Keene, J.D. 2001. Ribonucleoprotein infrastructure regulating the flow of genetic information between 
the genome and the proteome. Proc. Natl. Acad. Sci. USA 98:7018-7024.

6. Tuerk, C. and L. Gold. 1990. Systematic evolution of ligands by exponential enrichment: RNA ligands to bacteriophage T4 DNA polymerase. Science 249:505-510.

7. Zimmermann, B., I. Bilusic, C. Lorenz, and R. Schroeder. 2010. Genomic SELEX: a discovery tool for genomic aptamers. Methods 52:125-132.

8. McKeague, M. and M.C. Derosa. 2012. Challenges and opportunities for small molecule aptamer development. J. Nucleic Acids 2012:748913.

9. Svobodová, M., A. Pinto, P. Nadal, and C.K. O'Sullivan. 2012. Comparison of different methods for generation of single-stranded DNA for SELEX processes. Anal. Bioanal. Chem. 404:835-842.

10. Sugimoto, Y., J. Konig, S. Hussain, B. Zupan, T. Curk, M. Frye, and J. Ule. 2012. Analysis of CLIP and ICLIP methods for nucleotide-resolution studies of protein-RNA interactions. Genome Biol. 13:R67.

11. Ule, J., K.B. Jensen, M. Ruggiu, A. Mele, A. Ule, and R.B. Darnell. 2003. CLIP identifies Novaregulated RNA networks in the brain. Science 302:1212-1215.

12. Zhang, C. and R.B. Darnell. 2011. Mapping in vivo protein-RNA interactions at single-nucleotide resolution from HITS-CLIP data. Nat. Biotechnol. 29:607-614.

13. Hafner, M., M. Landthaler, L. Burger, M. Khorshid, J. Hausser, P. Berninger, A. Rothballer, M. Ascano, Jr., et al. 2010. Transcriptome-wide identification of RNA-binding protein and microRNA target sites by PAR-CLIP. Cell 141:129-141.

14. König, J., K. Zarnack, G. Rot, T. Curk, M. Kayikci, B. Zupan, D.J. Turner, N.M. Luscombe, and J. Ule. 2010. iCLIP reveals the function of hnRNP particles in splicing at individual nucleotide resolution. Nat. Struct. Mol. Biol. 17:909-915.

15. Licatalosi, D.D., A. Mele, J.J. Fak, J. Ule, M. Kayikci, S.W. Chi, T.A. Clark, A.C. Schweitzer, et al. 2008. HITS-CLIP yields genome-wide insights into brain alternative RNA processing. Nature 456:464469.

16. Moore, M.J., C. Zhang, E.C. Gantman, A. Mele, J.C. Darnell, and R.B. Darnell. 2014. Mapping Argonaute and conventional RNA-binding protein interactions with RNA at single-nucleotide resolution using HITS-CLIP and CIMS analysis. Nat. Protoc. 9:263-293.

17. Jangi, M., P.L. Boutz, P. Paul, and P.A. Sharp. 2014. Rbfox 2 controls autoregulation in RNA-binding protein networks. Genes Dev. 28:637-651.

18. Yeo, G.W., N.G. Coufal, T.Y. Liang, G.E. Peng, X.D. Fu, and F.H. Gage. 2009. An RNA code for the FOX2 splicing regulator revealed by mapping RNA-protein interactions in stem cells. Nat. Struct. Mol. Biol. 16:130-137.

19. Calvio, C., G. Neubauer, M. Mann, and A.I. Lamond. 1995. Identification of hnRNP P2 as TLS/ FUS using electrospray mass spectrometry. RNA 1:724-733.

20. Ajuh, P., B. Kuster, K. Panov, J.C. Zomerdijk, M. Mann, and A.I. Lamond. 2000. Functional analysis of the human CDC5L complex and identification of its components by mass spectrometry. EMBO J. 19:6569-6581.

21. Meissner, M., T. Dechat, C. Gerner, R. Grimm, R. Foisner, and G. Sauermann. 2000. Differential nuclear localization and nuclear matrix association of the splicing factors PSF and PTB. J. Cell. Biochem. 76:559-566.

22. Patton, J.G., E.B. Porro, J. Galceran, P. Tempst, and B. Nadal-Ginard. 1993. Cloning and charac- terization of PSF, a novel pre-mRNA splicing factor. Genes Dev. 7:393-406.

23. Peng, R., B.T. Dye, I. Perez, D.C. Barnard, A.B. Thompson, and J.G. Patton. 2002. PSF and p54nrb bind a conserved stem in U5 snRNA. RNA 8:1334-1347.

24. Stelzl, U., U. Worm, M. Lalowski, C. Haenig, F.H. Brembeck, H. Goehler, M. Stroedicke, M. Zenkner, et al. 2005. A human protein-protein interaction network: a resource for annotating the proteome. Cell 122:957-968.

25. Elliott, D.J. and P. Rajan. 2010. The role of the RNA-binding protein Sam68 in mammary tumourigenesis. J. Pathol. 222:223-226.

26. Lerga, A., M. Hallier, L. Delva, C. Orvain, I. Gallais, J. Marie, and F. Moreau-Gachelin. 2001. Identification of an RNA binding specificity for the potential splicing factor TLS. J. Biol. Chem. 276:6807-6816.

27. Galarneau, A. and S. Richard. 2009. The STAR RNA-binding proteins GLD-1, QKI, SAM68 and SLM-2 bind bipartite RNA motifs. BMC Mol. Biol. $10: 47$.

28. Karve, T.M. and A.K. Cheema. 2011. Small changes huge impact: the role of protein posttranslational modifications in cellular homeostasis and disease. J. Amino Acids 2011:207691.

29. Martin, L., M. Meier, S.M. Lyons, R.V. Sit, W.F. Marzluff, S.R. Quake, and H.Y. Chang. 2012.
Systematic reconstruction of RNA functional motifs with high-throughput microfluidics. Nat. Methods 9:1192-1194.

30. Ray, D., H. Kazan, E.T. Chan, L. Pena Castillo, S. Chaudhry, S. Talukder, B.J. Blencowe, Q. Morris, and T.R. Hughes. 2009. Rapid and systematic analysis of the RNA recognition specificities of RNA-binding proteins. Nat. Biotechnol. 27:667-670.

31. Buenrostro, J.D., C.L. Araya, L.M. Chircus, C.J. Layton, H.Y. Chang, M.P. Snyder, and W.J. Greenleaf. 2014. Quantitative analysis of RNAprotein interactions on a massively parallel array reveals biophysical and evolutionary landscapes. Nat. Biotechnol. 32:562-568.

32. Lambert, N., A. Robertson, M. Jangi, S McGeary, P.A. Sharp, and C.B. Burge. 2014. RNA Bind-n-Seq: quantitative assessment of the sequence and structural binding specificity of RNA-binding proteins. Mol. Cell 54:887-900.

Received 21 January 2017; accepted 12 May 2017.

Address correspondence to Kee K. Kim, Department of Biochemistry, College of Natural Sciences, Chungnam National University, Daejeon 34134, Republic of Korea. E-mail: kimkk@cnu.ac.kr

To purchase reprints of this article, contact: biotechniques@fosterprinting.com

\section{BEAD RUPTOR ELITE Bead Mill Homogenizer}

\section{Because Results Matter}
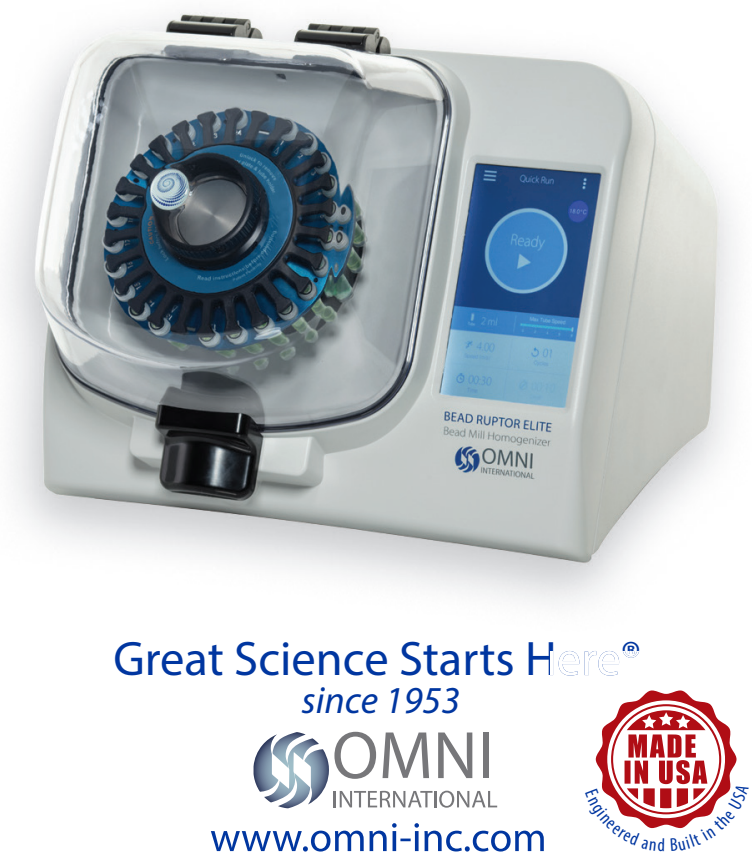